\title{
Quadruple-Junction Inverted Metamorphic Concentrator Devices
}

\author{
Ryan M. France, John F. Geisz, Iván García, Myles A. Steiner, William E. McMahon, Daniel J. Friedman, \\ Tom E. Moriarty, Carl Osterwald, J. Scott Ward, Anna Duda, Michelle Young, and Waldo J. Olavarria
}

\begin{abstract}
We present results for quadruple-junction inverted metamorphic (4J-IMM) devices under the concentrated direct spectrum and analyze the present limitations to performance. The devices integrate lattice-matched subcells with rear heterojunctions, as well as lattice-mismatched subcells with low threading dislocation density. To interconnect the subcells, thermally stable lattice-matched tunnel junctions are used, as well as a metamorphic GaAsSb/GaInAs tunnel junction between the lattice-mismatched subcells. A broadband antireflection coating is used, as well as a front metal grid designed for high concentration operation. The best device has a peak efficiency of $(43.8 \pm 2.2) \%$ at 327 -sun concentration, as measured with a spectrally adjustable flash simulator, and maintains an efficiency of $(42.9 \pm 2.1) \%$ at 869 suns, which is the highest concentration measured. The $V_{o c}$ increases from $3.445 \mathrm{~V}$ at 1 -sun to $4.10 \mathrm{~V}$ at 327 -sun concentration, which indicates high material quality in all of the subcells. The subcell voltages are analyzed using optical modeling, and the present device limitations and pathways to improvement are discussed. Although further improvements are possible, the 4J-IMM structure is clearly capable of very high efficiency at concentration, despite the complications arising from utilizing lattice-mismatched subcells.
\end{abstract}

Index Terms-Metamorphic, multijunction, III-V.

\section{INTRODUCTION}

$\mathbf{R}$ AISING the efficiency of multijunction solar cells can significantly lower the cost of electricity in a concentrator photovoltaic system. Because quadruple junction devices have the potential to achieve higher efficiency than state-ofthe-art triple junction devices, several laboratories are pursuing this design. However, integrating materials with the optimal bandgap combinations into a two-terminal device is challenging, particularly when the desired materials do not have the same lattice constant. Structural defects are created upon their integration and, if not properly controlled, significantly lower

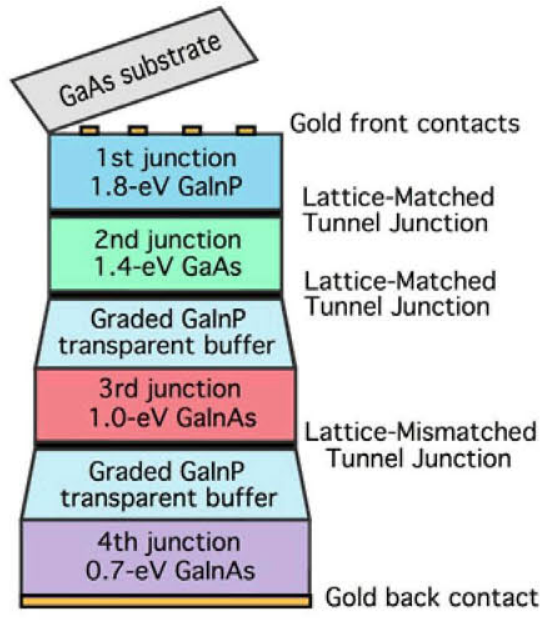

Fig. 1. General structure of the 4J-IMM. The width of the structure indicates the difference in lattice constant between the different layers. The GaAs substrate is removed after growth, allowing light access to the GaInP subcell first.

the device performance. One technique to combine materials with dissimilar lattice constants is wafer bonding. Using this approach, two independent epitaxial growths on two substrates with different lattice constants allow all subcells to be grown lattice-matched and dislocation-free [1], [2]. Then, the subcells are bonded together, limiting the structural defects to the inactive bonded interface. Another technique is to utilize compositionally graded buffers between subcells with different lattice constants, known as the "metamorphic" technique. In this case, the inactive graded buffers confine the structural defects, allowing a high-performance monolithic device. Quadruple junction devices have been previously demonstrated using the inverted metamorphic multijunction approach [3]-[6]. This approach allows the high-bandgap high-power-producing subcells to remain lattice-matched, while having bandgap flexibility in the lower bandgap subcells through the use of compositionally graded buffers. Triple-junction inverted metamorphic (3J-IMM) devices have achieved high efficiencies under concentration [7], but reports to date on quadruple-junction inverted metamorphic (4J-IMM) devices mainly focus on 1-sun space applications.

In this paper, we show the performance of the 4J-IMM device at 1-sun and under concentrated light. Our recent work on understanding and improving the subcell device structure and luminescent coupling between subcells has led to record performing GaInP junctions and GaInP/GaAs tandems, and work on understanding and controlling dislocation behavior in the graded buffers has led to lattice-mismatched bottom cells with only minor losses. We show the integration of these subcells 
into a full 4J-IMM device and analyze subcell data to determine the present limitations to performance and pathways to higher efficiency.

\section{EXPERIMENTAL PROCEDURES}

4J-IMM devices were grown using atmospheric-pressure metal-organic vapor phase epitaxy on $\left(\begin{array}{lll}0 & 0 & 1\end{array}\right)$ GaAs substrates miscut $2^{\circ}$ toward $\left(\begin{array}{lll}1 & 1 & 1\end{array}\right) \mathrm{B}$. The device structure is illustrated in Fig. 1. First, the lattice-matched 1.82-eV GaInP and 1.41-eV GaAs subcells were grown. These subcells have rear heterojunctions, which have been demonstrated to reduce nonradiative recombination [8]. Then, compositionally graded GaInP buffers were used to access lattice-mismatched 1.04- and $0.74-\mathrm{eV}$ GaInAs subcells. The dislocation density in the subcells is reduced by controlling the growth conditions [9] and structure [10] of the graded buffer, which limits dislocation formation due to composition variations, roughness, and atomic ordering [11], [12]. We have previously described the growth and properties of all individual subcells and graded buffers, and also two-junction and three-junction devices containing similar materials [8]-[10], [13], [14]. These subcells were interconnected using thermally stable lattice-matched tunnel junctions [15] and a metamorphic GaAsSb:C/GaInAs:Se tunnel junction between the two lattice-mismatched subcells [16]. After growth, the samples were bonded to a silicon handle, the substrate was removed, the cells were isolated, and a four-layer $\mathrm{MgF}_{2} / \mathrm{ZnS} / \mathrm{MgF}_{2} / \mathrm{ZnS}$ antireflection coating (ARC) was thermally evaporated.

Certified 1 -sun $I-V$ measurements were taken with a 1 -sun multisource simulator (OSMSS), with nine adjustable spectral zones. Certified $I-V$ measurements under concentrated light were taken at the NREL with an adjustable flash simulator, a tunable high intensity pulsed solar simulator (T-HIPSS), along with a spectral correction procedure to adjust data when the concentrator spectrum results in incorrect photocurrent balancing. This measurement technique reduces errors in the concentrator measurements due to the spectrum, which have been shown to result in erroneously high voltages, fill factors, and efficiencies [17]. Here, the first, second, and fourth junctions were filled correctly with $<1 \%$ error, but the third subcell was overfilled by $30 \%$; therefore, the correction procedure described in [18] was used. The 1 -sun subcell $J-V \mathrm{~S}$ and luminescent coupling parameters were extracted using a spectrally adjustable solar simulator using a method described elsewhere [19]. Spectrally resolved electroluminescence (EL) was measured in the dark over a range of injection current densities using a Spectral Evolution spectroradiometer [8]. External quantum efficiency (EQE) and reflection were measured on a custom-built instrument equipped with adjustable light-emitting diodes and voltage biasing capability. The junction bandgaps were determined using a fit to the EQE. All measurements were taken at $25^{\circ} \mathrm{C}$ on temperature-controlled stages.

\section{RESULTS}

\section{A. Concentration Performance}

The $I-V$ characteristics of the four-junction device are shown in Fig. 2, as a function of concentration. At 1 sun, the device

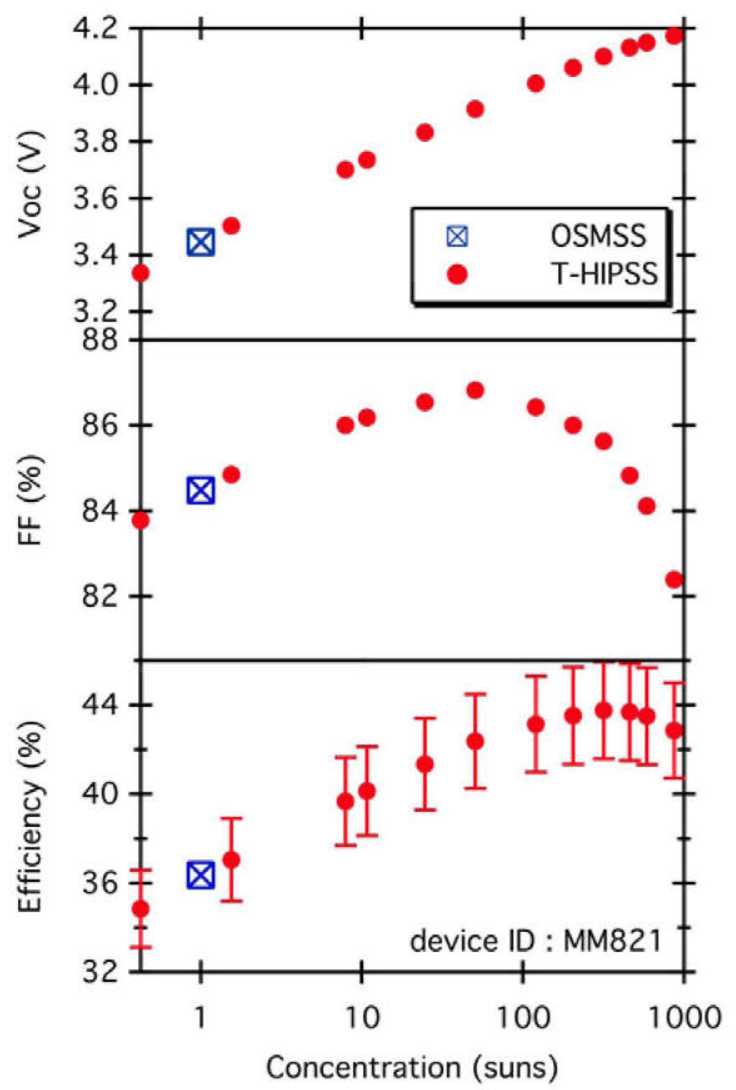

Fig. 2. Voltage, fill factor, and efficiency of the 4J-IMM device. The peak efficiency is $43.8 \%$ at 327 suns and is $42.9 \%$ at 867 suns. Results are independently certified by NREL, using an OSMSS 1-sun simulator and a T-HIPSS concentrator simulator.

has a $V_{\mathrm{oc}}$ of $3.445 \mathrm{~V}$, a $J_{\mathrm{sc}}$ of $12.49 \mathrm{~mA} / \mathrm{cm}^{2}$, and an efficiency of $36.35 \%$, as measured by the OSMSS. Notably, the T-HIPSS also provides data near 1-sun concentration, which agrees well with the OSMSS measurement due to the improved spectrum and measurement procedure [18]. At 327 suns, the efficiency peaks at $(43.8 \pm 2.2) \%$ and the $V_{\text {oc }}$ is $4.10 \mathrm{~V}$. The relative error of $5 \%$ is characteristic of the measurement and instrumentation. The series resistance losses begin to outweigh the additional voltage gains at higher concentrations, but the efficiency is still $(42.9 \pm 2.1) \%$ at 869 suns. These efficiencies are among the highest measured for a multijunction solar cell, proving the capability of the 4J-IMM structure under concentration.

Series resistance is the single greatest limitation to the performance of this device at high concentration, as is clearly evident in the low fill factor under concentrated light. Both the frontcontact resistance and top cell sheet resistance limit the performance, as determined from transmission-line measurements. However, the sheet resistance of the top cell has been reduced from 400 to $100 \Omega / \square$ by using a rear-heterojunction design with a thick emitter layer rather than a traditional front-homojunction design [8], [20]. The rear-heterojunction structure allows the full thickness of the top cell to transport carriers laterally. The resistivity through the front contact and window layer is $3 \times$ $10^{-4} \Omega \cdot \mathrm{cm}^{2}$, which results in high $I^{2} R$ loss at high current densities. Investigation and reduction of the source of this resistivity is necessary for higher efficiency at concentration. 
TABLE I

BANDGAP $\left(E_{g}\right)$, Voltage IN THE DETAILED BALANCE LiMIT $\left(V_{\mathrm{d}} \mathrm{b}\right)$, EXTERNAL RADIATIVE EFFICIENCY $\left(\eta_{\text {ext }}\right)$, OPEN-CiRCUIT VOLTAGE $\left(V_{o c}\right)$, AND BANDGAP-VOLTAGE OFFSET ( $W_{O c}$ ) OF EACH SUBCELL, DETERMINED FROM EQE AND EL

\begin{tabular}{lccccc}
\hline \hline Junction & $E_{g}(\mathrm{eV})$ & $V_{\mathrm{db}}(\mathrm{V})$ & $\eta_{\mathrm{ext}}(\%)$ & $V_{\mathrm{oc}}(\mathrm{V})$ & $W_{\mathrm{oc}}(\mathrm{V})$ \\
\hline 1 & 1.82 & 1.53 & 2.8 & 1.43 & 0.39 \\
2 & 1.41 & 1.12 & 1.8 & 1.02 & 0.39 \\
3 & 1.04 & 0.78 & 1.6 & 0.67 & 0.37 \\
4 & 0.74 & 0.48 & 0.4 & 0.34 & 0.40 \\
\hline \hline
\end{tabular}

\section{B. Subcell Nonradiative Recombination Analysis}

To analyze nonradiative recombination, we analyze the subcell voltage loss using different voltage-based metrics. The subcell voltages are determined from the reciprocity theorem described by Rau [21], using $V(J)=V_{\mathrm{db}}(J)+V_{T} \ln \left(\eta_{\text {ext }}\right)$, where $V_{T}=k T / q$ is the thermal voltage. Here, $V_{\mathrm{db}}$ is the subcell voltage in the detailed balance limit, which is determined from the solar spectrum and the EQE of each subcell, corrected for luminescent coupling effects [22]. $\eta_{\text {ext }}$ is the external radiative efficiency, measured with EL using $\eta_{\text {ext }}=$ $J_{\text {emitted }} / J_{\text {injected, }}$, where the injected currents to each subcell are corrected for luminescent coupling [19]. The bandgapvoltage offset, i.e., $W_{\mathrm{oc}}\left(=E_{g} / q-V_{\mathrm{oc}}\right)$, is commonly used as a metric of material quality across solar cells with different bandgaps and is listed in Table I along with the subcell $V_{\mathrm{Oc}}$ and $V_{\mathrm{db}}$ calculations. The $W_{\mathrm{oc}}$ of each junction is $0.4 \mathrm{~V}$ or less, indicating good material quality despite the lattice-mismatch in the lower bandgap subcells [23]. This metric changes with bandgap [23]. Lower $W_{\mathrm{OC}} \mathrm{s}$ are expected for lower bandgap materials, and therefore, the fourth subcell apparently has the lowest material quality of the four subcells. However, this subcell is $3.8 \%$ lattice-mismatched to the GaAs substrate and still has higher $V_{\mathrm{oc}}$ and lower $W_{\mathrm{oc}}$ than germanium solar cells (from [24], Ge $V_{\mathrm{oc}}=0.24 \mathrm{~V}, W_{\mathrm{oc}}=0.43 \mathrm{~V}$ ), which are commonly used as bottom cells of multijunction solar cells and have a similar bandgap.

The difference between $V_{\mathrm{oc}}$ and $V_{\mathrm{db}}$, termed $W_{\mathrm{db}}(=$ $\left.V_{T} \ln \left(\eta_{\text {ext }}\right)\right)$, has also been used to describe voltage loss and is less dependent on bandgap and thickness [22]. However, this metric combines voltage loss from nonradiative recombination within the subcell, termed internal loss, with voltage loss from radiated light that escapes the active layer of the subcell and is absorbed elsewhere in the multijunction structure, which is termed optical loss. Optical modeling of the multijunction structure can separate these loss mechanisms. A description of the technique and assumptions are given in [13], and errors are not further addressed here. The probabilities that radiated light is reabsorbed within the subcell $\left(\overline{P_{\mathrm{abs}}}\right)$ or escapes out the front surface of the solar cell structure $\left(\overline{P_{\text {esc }}}\right)$ are determined using a ray-optic model of photon propagation and the transfer matrix method to calculate reflection and transmission probabilities [13]. The total external radiative efficiency $\eta_{\text {ext }}$ is then

$$
\eta_{\text {ext }}=\frac{\eta_{\text {int }} \overline{P_{\mathrm{esc}}}}{1-\eta_{\text {int }} \overline{P_{\mathrm{abs}}}} .
$$

TABLE II

Probability That a Radiated Photon Escapes the Multiunction

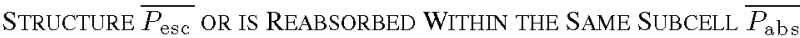
DETERMINED BY OPTTCAL MODELING

\begin{tabular}{lccccc}
\hline \hline Junction & $\overline{P_{\mathrm{esc}}}(\%)$ & $\overline{P_{\text {abs }}}(\%)$ & $\eta_{\text {int }}(\%)$ & $W_{\text {opt }}(\mathrm{mV})$ & $W_{\text {int }}(\mathrm{mV})$ \\
\hline 1 & 1.3 & 69 & 87 & 80 & 20 \\
2 & 0.8 & 87 & 76 & 70 & 30 \\
3 & 0.7 & 89 & 73 & 70 & 40 \\
4 & 1.1 & 96 & 25 & 30 & 110 \\
\hline \hline
\end{tabular}

These parameters are used to evaluate the voltage loss from nonradiative recombination within the subcell $W_{\text {int }}$ or due to the loss of radiated photons elsewhere in the multijunction structure $W_{\text {opt }}$ at 1 -sun current density. The internal radiative efficiency $\eta_{\text {int }}$ can also be determined.

The subcell internal radiative efficiency $\eta_{\text {int }}$ describes the fraction of the total recombination within the subcell that is radiative and may vary with current density. Equation (1) allows the voltage loss with respect to the detailed balance limit $W_{\mathrm{db}}$ to be separated into optical loss $W_{\text {opt }}$ and internal loss $W_{\text {int }}$. $W_{\text {opt }}$ describes the voltage loss from radiated light that is reabsorbed within the multijunction structure when the subcell has no nonradiative recombination, $\eta_{\text {int }}=1$, and $W_{\text {int }}$ is related to the nonradiative losses within the subcell

$$
\begin{aligned}
W_{\mathrm{db}} & =V_{T} \ln \left(\eta_{\mathrm{ext}}\right)=W_{\mathrm{opt}}+W_{\mathrm{int}} \\
W_{\mathrm{opt}} & =W_{\mathrm{db}}\left(\eta_{\mathrm{int}}=1\right)=V_{T} \ln \left(\frac{\overline{P_{\mathrm{esc}}}}{1-\overline{P_{\mathrm{abs}}}}\right) \\
W_{\mathrm{int}} & =V_{T} \ln \left(\eta_{\mathrm{ext}} \frac{1-\overline{P_{\mathrm{abs}}}}{\overline{P_{\mathrm{esc}}}}\right) \\
& =V_{T} \ln \left(\eta_{\mathrm{int}} \frac{1-\overline{P_{\mathrm{abs}}}}{1-\eta_{\mathrm{int}} \overline{P_{\mathrm{abs}}}}\right) .
\end{aligned}
$$

The modeled values of $\overline{P_{\text {esc }}}$ and $\overline{P_{\text {abs }}}$ for each junction are shown in Table II, along with the voltage loss metrics evaluated at the 1-sun current density of this device. The optical losses in the top three junctions dominate the total voltage loss with respect to the detailed balance limit. Interestingly, the fourth subcell has a lower optical loss than the other subcells because of the gold contact, which serves as a back reflector (see Fig. 1) and raises $\overline{P_{\mathrm{abs}}}$. The top cell is thinner than the other cells, which lowers $\overline{P_{\mathrm{abs}}}$ and raises $W_{\text {opt }}$. These optical losses are largely inherent to the geometry of this multijunction structure and are not easily reduced without the introduction of wavelengthspecific reflectors within the structure [25]. In addition, some of this loss is recovered by transferring energy to other subcells via luminescent coupling, which is not described in this analysis [26].

The voltage loss from internal nonradiative recombination within the top three subcells is very low at 1 sun, and the bottom cell is also good considering the large amount of lattice mismatch. $W_{\text {int }}$ is determined at higher concentrations by evaluating the EL from higher injection currents, as shown in Fig. 3. We assume that the probabilities $\overline{P_{\text {abs }}}$ and $\overline{P_{\text {esc }}}$ do not vary with current density, and therefore, $W_{\text {opt }}$ does not change with concentration. The voltage loss due to nonradiative recombination 


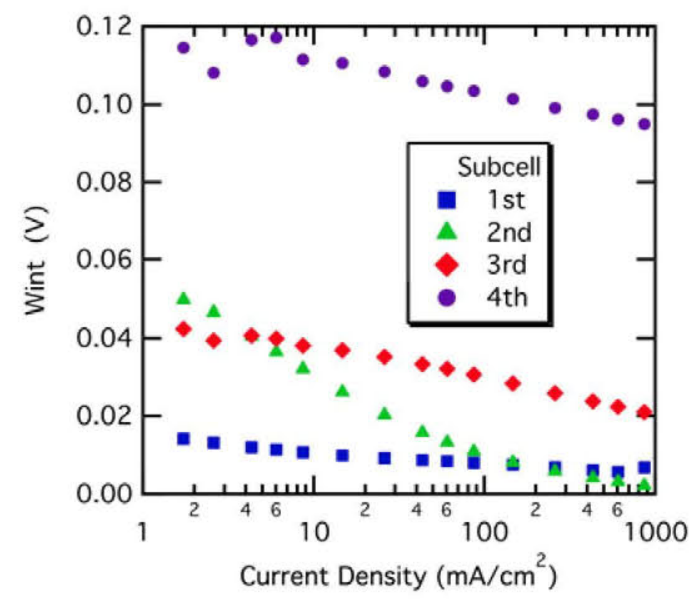

Fig. 3. Voltage loss due to nonradiative recombination within each subcell $W_{\text {int }}$ determined from EL and optical modeling. The maximum measured current density is the equivalent of 80 -sun concentration for this device.

in the lattice-matched subcells becomes negligible at higher concentration, in part due to the exceptionally high-quality rearheterojunction top cell. Further reductions in nonradiative recombination are not necessary in these subcells. The voltage loss in the third subcell reduces to $20 \mathrm{mV}$ at $1000 \mathrm{~mA} / \mathrm{cm}^{2}$ (the equivalent of 80 suns for this device) and likely further decreases at higher concentrations. The low loss in the third subcell, which is $1.9 \%$ mismatched to the GaAs substrate, demonstrates the performance capability of these metamorphic GaInAs subcells. The total voltage loss due to nonradiative recombination in the 4J-IMM is very small, i.e., $120 \mathrm{mV}$ at 80 suns. However, some voltage gain is still achievable, particularly in the fourth subcell. Further effort to reduce nonradiative recombination in this $4 \mathrm{~J}$ IMM should be directed toward improving the material quality of the fourth subcell and second graded buffer.

\section{Carrier Collection and Bandgap Combination Analysis}

Fig. 4 shows the luminescent-coupling-corrected EQE and IQE, reflection of the four-junction 1.82/1.41/1.04/0.74-eV device, and subcell photocurrents determined by a luminescent coupling analysis [19]. The photocurrents are nearly current matched, and the limiting current determined from the luminescent coupling analysis matches well with the $J_{\mathrm{sc}}$ from the official measurement. Some carrier collection losses still exist, including losses within the semiconductor and from the frontreflection. As is evident in the cumulative IQE, the collection is imperfect on both the short- and long-wavelength side of the top cell, presumably due to parasitic absorption in the window and tunnel junction, respectively. However, the collection in the lattice-mismatched subcells is good, indicating that the current is not significantly limited by the metamorphic nature of the IMM.

The front reflection of the grid fingers and ARC limits the photocurrent produced by each subcell. The total current loss across the usable spectrum of this device due to reflection is $>2 \mathrm{~mA} / \mathrm{cm}^{2}$ from grid shadowing, and also $>2 \mathrm{~mA} / \mathrm{cm}^{2}$ from the ARC, determined by integrating the reflection with the direct spectrum. Some reflection is unavoidable. However, evenly
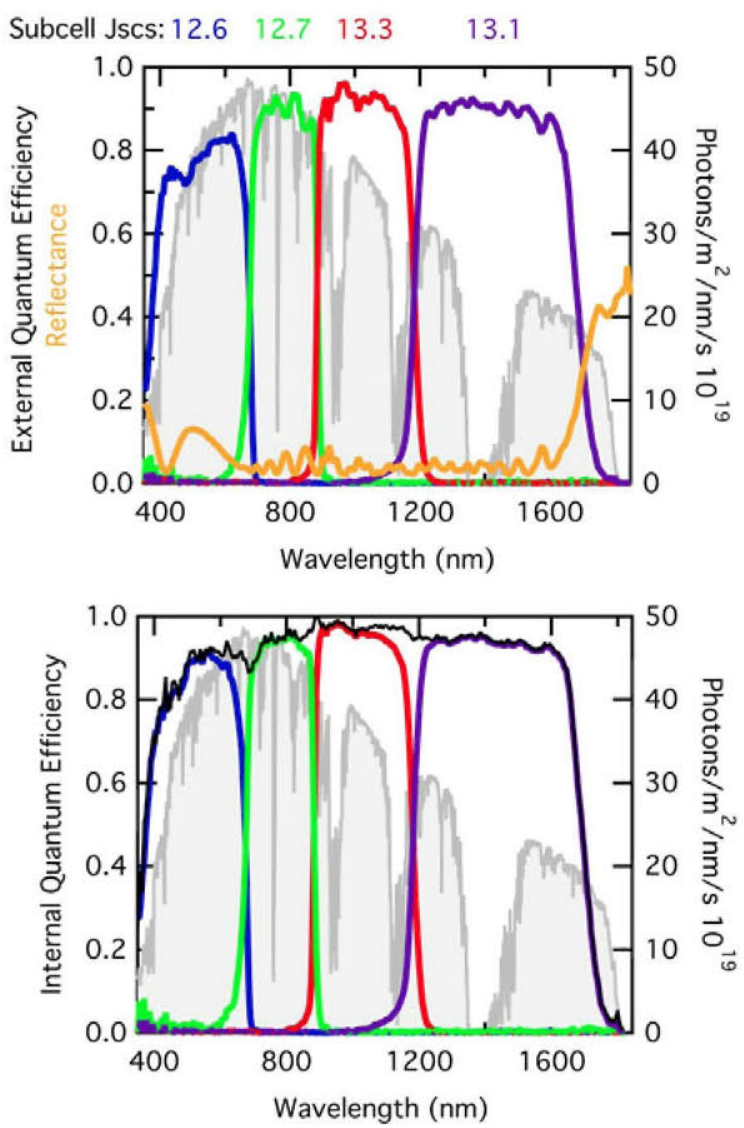

Fig. 4. (Top) EQE and reflection of the 4J-IMM, corrected for luminescent coupling. The estimated 1-sun currents $\left(\mathrm{mA} / \mathrm{cm}^{2}\right)$ are included above each respective junction's photoresponse, calculated using an analysis of the luminescent coupling parameters. (Bottom) IQE of the $4 \mathrm{~J}-\mathrm{IMM}$, corrected for luminescent coupling. The black line is the cumulative IQE. The direct spectrum is displayed in both figures for reference.

distributing $1 \mathrm{~mA} / \mathrm{cm}^{2}$ over the four junctions should increase the absolute efficiency by about $1 \%$, and therefore, increasing the current by reducing front-surface reflection can substantially improve the device performance. This device has gridfinger widths of $8 \mu \mathrm{m}$ and spacings of $150 \mu \mathrm{m}$. Using thinner, high-aspect ratio, grid fingers for the same finger spacing would increase efficiency but requires higher conductivity through the contact and window layers.

The absorbed and utilized spectrum of this four-junction device extends from 350 to $1750 \mathrm{~nm}$, which is a larger portion of the spectrum than in GaInP/GaAs/GaInAs three-junction devices. A four-layer $\mathrm{ARC}$ is used to minimize reflection. The $\mathrm{ARC}$ is comprised of alternating layers of high- and low-index dielectrics, forming a so-called Herpin equivalent of a threelayer ARC with an intermediate index middle layer [27]. This $\mathrm{ARC}$ is optimal for this device structure but still sacrifices light between 400 and $700 \mathrm{~nm}$. By design, the GaInP subcell receives more light than the other junctions; therefore, this reflection only lowers the GaInP photocurrent slightly below the GaAs photocurrent. However, if this light were instead absorbed, it would still benefit the multijunction device through luminescent coupling from the top subcell to lower subcells [28]. In addition, the bandgap combination or subcell thicknesses could 


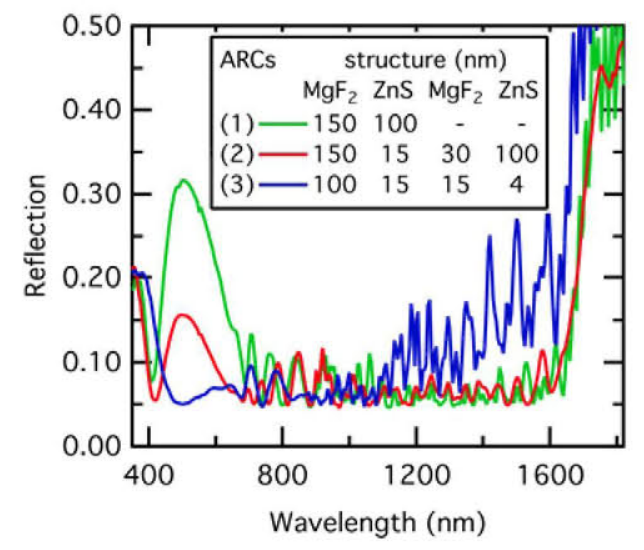

Fig. 5. Measured reflection of 4J-IMMs with different ARCs. The material combination and nominal thicknesses of the ARCs are included. About $5 \%$ of the reflection is due to the front grids, and the rest is from the imperfect ARC.

be reoptimized to evenly distribute the light, potentially raising both the current and voltage of the device.

While less reflective ARCs are always desirable, ARCs with less total reflected power are attainable by tuning the layer thicknesses and reducing the reflectance in the top subcell at the expense of the fourth subcell. Fig. 5 shows the reflectance of different ARCs measured on 4J-IMM structures and the nominal thickness of the ARC layers. Two different four-layer ARCs are shown as well as a two-layer ARC optimized for this device structure to show the benefit of using a four-layer ARC. The difference in reflected power from the 1-sun AM1.5 direct spectrum between the two four-layer ARCs, calculated by integrating with the direct spectrum over the usable spectrum of this device, is $>10 \mathrm{~W} / \mathrm{m}^{2}$, favoring the $\mathrm{ARC} 3$, which is reflected in the long-wavelength range. This increase is primarily due to differences in the energy of the reflected photons; the difference in the total number of reflected photons is low.

By changing from $\mathrm{ARC} 2$ to the $\mathrm{ARC} 3$, the cell loses longwavelength photons and gains an equal amount of shortwavelength photons. To benefit from this extra power, the bandgap combination should be changed. The top cell would benefit from a higher bandgap if the nonradiative recombination is kept low when making this change, which is not straightforward and requires research. To accommodate the loss of photons in the long-wavelength range, we note that an additional $1-2 \mathrm{~mA} / \mathrm{cm}^{2}$ can be gained if the photons below the bottom cell bandedge and above the absorption edge at $1800 \mathrm{~nm}$ in the direct spectrum are collected (shown in Fig 4). Lowering the bottom cell bandgap to $0.70-0.72 \mathrm{eV}$ (the optimum bandgap will depend on the operating temperature) would absorb these photons and is possible in this metamorphic device with additional research into the graded buffer. Using an existing ARC that reflects less power and making changes to the bandgap combination while maintaining current-matching and low nonradiative recombination is another pathway toward higher efficiency.

\section{CONCLUSION}

We have shown the performance of the 4J-IMM solar cell under concentrated light. Certified measurements taken using an adjustable flash simulator at the NREL show that the device efficiency peaks at $(43.8 \pm 2.2) \%$ at 327 suns and is $(42.9 \pm 2.1) \%$ at 869 suns, proving the capability of $4 \mathrm{~J}$-IMM concentrator cells. The $V_{\text {oc }}$ is high, $3.445 \mathrm{~V}$ at 1 -sun, indicating low nonradiative recombination and good material quality in all subcells. We isolate the subcell voltage loss due to nonradiative recombination within the subcell by optical modeling. While the total nonradiative loss is low, lowering nonradiative recombination in the fourth subcell would lead to voltage increases. Tuning the bandgap combination by changing the top and bottom cell bandgaps while using a different ARC can lead to increases in both current and voltage. Major increases to the fill factor at high concentrations are possible if series resistance through the front contact and window layer is reduced. This 4J-IMM structure already performs very well and is not severely hindered by the lattice-mismatched subcells. Even so, significant efficiency gains are possible for this device with additional research into lowering series resistance, reducing front-surface reflections, raising the top cell bandgap without sacrificing material quality, and improving and extending the graded buffer for the bottom cell.

\section{REFERENCES}

[1] P. T. Chiu, D. C. Law, R. L. Woo, S. B. Singer, D. Bhusari, W. D. Hong, A. Zakaria, J. Boisevert, S. Mesropian, R. R. King, and N. H. Karam, "Direct semiconductor bonded 5J cell for space and terrestrial applications," IEEE J. Photovoltaics, vol. 4, no. 1, pp. 493-497, Jan. 2014.

[2] F. Dimroth, M. Grave, P. Beutel, U. Fiedler, C. Karcher, T. N. D. Tibbits, E. Oliva, G. Siefer, M. Schachtner, A. Wekkeli, A. W. Bett, R. Krause, M. Piccin, N. Blanc, C. Drazek, E. Guiot, B. Ghyselen, T. Salvetat, A. Tauzin, T. Signamarcheix, A. Dobrich, T. Hannappel, and K. Schwarzburg, "Wafer bonded four-junction GaInP/GaAs/GaInAsP/ GaInAs concentrator solar cells with $44.7 \%$ efficiency," Prog. Photovoltaics, vol. 22, pp. 227-282 2014.

[3] M. Stan, D. Aiken, B. Cho, A. Cornfeld, V. Ley, P. Patel, P. Sharps, and T. Varghese, "High-efficiency quadruple junction solar cells using OMVPE with inverted metamorphic device structures," J. Cryst. Growth, vol. 312, pp. 1370-1374, 2010.

[4] A. B. Cornfeld, D. Aiken, B. Cho, A. V. Ley, P. Sharps, M. Stan, and T. Varghese, "Development of a four sub-cell inverted metamorphic multijunction (IMM) highly efficient AM0 solar cell," in Proc. Photovoltaic Spec. Conf., Honolulu, HI, USA, 2010, pp. 105-109.

[5] P. Patel, D. Aiken, A. Boca, B. Cho, D. Chumney, M. B. Clevenger, A. Cornfeld, N. Fatemi, Y. Lin, J. McCarty, F. Newman, P. Sharps, J. Spann, M. Stan, J. Steinfeldt, C. Strautin, and T. Varghese, "Experimental results from performance improvement and radiation hardening of inverted metamorphic solar cells," IEEE J. Photovoltaics, vol. 2, no. 3, pp. 377-381, Jul. 2012.

[6] D. J. Friedman, J. F. Geisz, A. G. Norman, M. W. Wanlass, and S. R. Kurtz, "0.7-eV GaInAs junction for a GaInP/GaAs/GaInAs(1eV)/ GaInAs $(0.7 \mathrm{eV})$ four-junction solar cell," in Proc. 4th World Conf. Photovoltaic Energy Convers., Waikoloa, HI, USA, 2006, pp. 598-602.

[7] J. F. Geisz, S. R. Kurtz, M. W. Wanlass, J. S. Ward, A. Duda, D. J. Friedman, J. M. Olson, W. E. McMahon, T. Moriarty, and J. Kiehl, "High-efficiency GaInP/GaAs/InGaAs triple-junction solar cells grown inverted with a metamorphic bottom junction," Appl. Phys. Lett., vol. 91, pp. 023502-1-023502-3, 2007.

[8] J. F. Geisz, M. A. Steiner, I. Garcıa, S. R. Kurtz, and D. J. Friedman, "Enhanced external radiative efficiency for $20.8 \%$ efficient single-junction GaInP solar cells," Appl. Phys. Lett., vol. 103, pp. 041118-1-041118-5, 2013.

[9] R. M. France, J. F. Geisz, M. A. Steiner, B. To, M. J. Romero, W. J. Olavarria, and R. R. King, "Reduction of crosshatch roughness and threading dislocation density in metamorphic GaInP buffers and GaInAs solar cells," J. Appl. Phys., vol. 111, pp. 103528-1-103528-7, 2012.

[10] R. M. France, I. Garcia, W. E. McMahon, A. G. Norman, J. Simon, J. Geisz, D. J. Friedman, and M. J. Romero, "Lattice-mismatched 0.7-eV GaInAs solar cells grown on GaAs using GaInP compositionally graded buffers," IEEE J. Photovoltaics, vol. 4, no. 1, pp. 190-195, Jan. 2013. 
[11] L. M. McGill, E. A. Fitzgerald, A. Y. Kim, J. W. Huang, S. S. Yi, P. N. Grillot, and S. A. Stockman, "Microstructural defects in metalorganic vapor phase epitaxy of relaxed, graded InGaP: Branch defect origins and engineering," J. Vac. Sci. Technol. B, vol. 22, pp. 1899-2011, 2004.

[12] R. M. France, W. E. McMahon, A. G. Norman, J. F. Geisz, and M. J. Romero, "Control of misfit dislocation glide plane distribution during strain relaxation of CuPt-ordered GaInAs and GaInP," J. Appl. Phys., vol. 112, pp. 023520-1-023520-6, 2012.

[13] M. A. Steiner, J. F. Geisz, I. Garcia, D. J. Friedman, A. Duda, and S. R. Kurtz, "Optical enhancement of Voc in high quality GaAs solar cells," J. Appl. Phys., vol. 113, pp. 123109-1-123109-11, 2013.

[14] R. M. France, J. F. Geisz, M. A. Steiner, D. J. Friedman, J. S. Ward, J. M. Olson, W. Olavarria, M. Young, and A. Duda, "Pushing inverted metamorphic multijunction solar cells towards higher efficiency at realistic operating conditions," IEEE J. Photovoltaics, vol. 3, no. 2, pp. 893-898, Apr. 2013.

[15] I. Garcia, J. F. Geisz, R. M. France, M. A. Steiner, and D. J. Friedman, "Component integration strategies in metamorphic 4-junction III-V concentrator solar cells," presented at the Int. Conf. Concentrator Photovoltaic Syst., Albuquerque, NM, USA, 2014.

[16] I. Garcia, J. F. Geisz, R. M. France, J. Kang, S.-H. Wei, M. Ochoa, and D. J. Friedman, "Metamorphic Ga0.76In0.24As/GaAs0.75Sb0.25 tunnel junctions grown on GaAs substrates," vol. 116, pp. 074508-1-074508-16, 2014.

[17] C. R. Osterwald, M. W. Wanlass, T. Moriarty, M. A. Steiner, and K. A. Emery, "Concentrator cell efficiency measurement errors caused by unfiltered xenon flash solar simulators," presented at the Int. Conf. Concentrator Photovoltaic Syst., Albuquerque, NM, USA, 2014.

[18] C. R. Osterwald, M. W. Wanlass, T. Moriarty, M. A. Steiner, and K. A. Emery, "Empirical procedure to correct concentrator cell efficiency measurement errors caused by unfiltered xenon flash solar simulators," presented at the Photovoltaic Spec. Conf., Denver, CO, USA, 2014.

[19] J. F. Geisz, R. M. France, I. Garcia, M. A. Steiner, and D. J. Friedman, "Device characterization for design optimization of 4 junction inverted metamorphic concentrator solar cells," presented at the Int. Conf. Concentrator Photovoltaics Syst., Albuquerque, NM, USA, 2014.
[20] J. F. Geisz, M. A. Steiner, I. Garcia, R. M. France, D. J. Friedman, and S. R. Kurtz, "Implications of redesigned, high-radiative efficiency GaInP junctions on III-V multijunction concentrator solar cells," submitted for publication.

[21] U. Rau, "Reciprocity relation between photovoltaic quantum efficiency and electroluminescent emission of solar cells," Phys. Rev. B, vol. 76, pp. 085303-1-085303-8, 2007.

[22] E. S. Toberer, A. C. Tamboli, M. Steiner, and S. Kurtz, "Analysis of solar cell quality using voltage metrics," in Proc. Photovoltaics Spec. Conf., Austin, TX, USA, 2012, pp. 1327-1331.

[23] R. R. King, D. Bhusari, A. Boca, D. Larrabee, X.-Q. Liu, W. Hong, C. M. Fetzer, D. C. Law, and N. H. Karam, "Band gap-voltage offset and energy production in next-generation multijunction solar cells," Prog. Photovoltaics, vol. 19, pp. 797-812, 2010.

[24] D. J. Friedman and J. M. Olson, "Analysis of Ge junctions for GaInP/GaAs/Ge three-junction solar cells," Prog. Photovoltaics, vol. 9, pp. 179-189, 2001.

[25] I. Garcia, J. Geisz, M. Steiner, J. Olson, D. Friedman, and S. Kurtz, "Design of semiconductor-based back reflectors for high Voc monolithic multijunction solar cells," in Proc. Photovoltaic Spec. Conf., Austin, TX, USA, 2012, pp. 2042-2047.

[26] D. J. Friedman, J. F. Geisz, and M. A. Steiner, "Effect of luminescent coupling on the optical design of multijunction solar cells," IEEE J. Photovoltaics, vol. 4, no. 3, pp. 986-990, May 2014.

[27] D. J. Aiken, "High performance anti-reflection coatings for broadband multi-junction solar cells," Solar Energy Mater. Solar Cells, vol. 64, pp. $393-404,2000$.

[28] M. A. Steiner and J. F. Geisz, "Non-linear luminescent coupling in series-connected multijunction solar cells," Appl. Phys. Lett., vol. 100, pp. 251106-1-251106-5, 2012.

Authors' photographs and biographies not available at the time of publication. 